\title{
Investigating population risk factors of pancreatic cancer by evaluation of optical markers in the duodenal mucosa
}

\author{
Vladimir Turzhitsky ${ }^{\mathrm{a}, *}$, Yang Liu ${ }^{\mathrm{a}}$, Nahla Hasabou ${ }^{\mathrm{b}}$, Michael Goldberg ${ }^{\mathrm{b}}$, Hemant K. Roy ${ }^{\mathrm{b}}$, \\ Vadim Backman ${ }^{\mathrm{a}}$ and Randall Brand ${ }^{\mathrm{b}}$ \\ ${ }^{a}$ Biomedical Engineering Department, Northwestern University, Evanston, IL, USA \\ ${ }^{\mathrm{b}}$ Department of Internal Medicine, Evanston Northwestern Healthcare, Evanston, IL, USA
}

\begin{abstract}
Pancreatic cancer screening has been hampered by the high rate of complications associated with interrogating the pancreas. The closest non-invasively accessible mucosa available for pancreatic cancer screening is the periampullary duodenal tissue. Our earlier report has shown the potential of using optical markers to interrogate this tissue for the presence of pancreatic cancer. In this study, we report a larger data set of low-coherence enhanced backscattering (LEBS) and elastic light scattering fingerprinting (ELF) optical markers from the periampullary duodenal mucosa. Optical measurements from biopsy samples were acquired from a total of 203 patients with varying clinical classification including healthy controls, a family history of pancreatic cancer, pancreatitis, mucinous cystic precursor lesions, pancreatic cancer, and other pancreatic malignancies. Evaluation of the performance of an independent testing set for discriminating healthy control patients from pancreatic cancer patients showed a $95 \%$ sensitivity, $71 \%$ specificity, and $85 \%$ area under the receiver operator characteristic (AUROC) curve. Importantly, this performance was uncompromised for detecting potentially curable stages of the disease. Additionally, optical markers in higher risk populations such as family history and pancreatitis had values between those of healthy control and pancreatic cancer patients, thus allowing for future investigations of screening from these high risk groups.
\end{abstract}

Keywords: Pancreatic cancer screening, field effect, duodenum, backscattering spectroscopy

\section{Introduction}

Pancreatic cancer is one of the deadliest cancers with a 5 year survival rate of less than $5 \%$ and is the 4 th largest cause of cancer-related deaths in the United States [7]. The dismal survival rate is due in a large part to our inability to detect a pancreatic cancer at an early stage when cure is still possible. The clinical challenges that must be overcome in devising an early detection strategy for pancreatic cancer include the early spread of the disease, late onset of clinical symptom development and the inaccessible location of the

\footnotetext{
*Corresponding author. Vladimir Turzhitsky, Biomedical Engineering Department, Northwestern University, 2145 Sheridan Road, Evanston, IL 60208, USA. Tel.: +1 847491 7167; Fax: +1 847491 4928; E-mail: v-turzhitsky@northwestern.edu.
}

organ. Furthermore, it is not feasible to screen the general population for this cancer due to its low incidence. Thus, there is a need to identify a subset of the general population that has a higher prevalence of the disease in whom one could justify the cost and potential risks associated with an early detection strategy. Currently, individuals from pancreatic cancer-prone families are the only population subset in which the prevalence of the disease is great enough to warrant pancreatic cancer surveillance [1]. Unfortunately, even if an effective early detection approach is applied to these patients, only a minority of pancreatic cancer cases would be impacted because a maximum of only $10 \%$ of all diagnosed patients have a family history [24]. Despite this small contribution, screening this high risk group has the potential to save many lives. 
Pancreatic adenocarcinomas typically evolve from three types of precursor lesions: pancreatic intraepithelial neoplasia (PanIN), mucinous cystic neoplasms $(\mathrm{MCN})$, and intraductal papillary mucinous neoplasms (IPMN). Detection and removal of these precursor lesions has the potential to decrease the patient's risk of being diagnosed with this fatal disease $[3,21]$ despite the fact that most instances will never advance to a clinically significant cancer $[4,12]$. Because of the typically asymptomatic nature of these types of lesions, much of their current detection is being accomplished by accident [26]. Therefore, the need for an approach that can stratify the risk of patients with recognized pancreatic precursor lesions would be of great clinical value.

We have previously demonstrated that optical evaluation of the duodenal mucosa could accurately discriminate control patients from pancreatic adenocarcinoma patients. This approach was based on the premise that the optical changes in the periampullary duodenum were a result of the technology detecting the field effect [13]. The field effect is the concept that the genetic and environmental milieu that results in a neoplastic lesion is present and detectable in some form in nearby locations. This non-localized detection of neoplastic changes has been studied in lung cancer $[5,6$, $22]$, colon cancer [2,18], and more recently in pancreatic cancer $[13,16,17]$. The evaluation of periampullary duodenal tissue is a particularly attractive strategy for pancreatic cancer screening because the potentially serious complications of probing the pancreatic duct are circumvented.

The aim of this study was to extend the previously reported results by evaluating the same optical markers derived from low-coherence enhanced backscattering (LEBS) and four-dimensional elastic light-scattering fingerprinting (4D-ELF) on a larger data set. Additionally, we assessed the potential of this technique as a risk stratification tool by investigating patients with a family history of pancreatic cancer and mucinous cystic lesions (IPMNs and MCNs). We also determined whether benign inflammatory conditions of the pancreas could be discriminated from either pancreatic cancer or control patients.

\section{Methods}

\subsection{Patients}

Human samples were acquired in accordance with the institutional review board at Evanston Northwest- ern Healthcare. Two biopsies were acquired from the periampullary mucosa (1-3 cm from ampulla) after informed consent of the patient either during endoscopy or from surgically resected Whipple specimens within 30 minutes of the Whipple procedure. Biopsies were washed in phosphate buffered saline (PBS) and oriented on a glass slide such that the epithelial surface of the biopsy was illuminated. Biopsies were kept hydrated with PBS throughout the acquisition of optical measurements.

\subsection{Instrument}

The instrument used to collect LEBS and 4D-ELF signals was described in detail in our earlier publications $[8,10,13]$. Briefly, light from a 500 Watt xenon lamp (Oriel Instruments) was collimated and passed through a 4-f lens system, making the spatial coherence length $\sim 80 \mu \mathrm{m}$. The light is polarized with a linear polarizer, apertured to a $1 \mathrm{~mm}$ diameter spot size, and then passed through a beam splitter onto the sample at a $15^{\circ}$ angle of incidence with respect to the sample surface. Light scattered within $+/-7^{\circ}$ of the incident angle in the backward direction is reflected by the beam splitter and collected by a lens $(\mathrm{f}=31 \mathrm{~mm})$. An analyzer is used to distinguish between the co-polarized ( $\mathrm{I}_{\|}, 0^{\circ}$ with respect to the incident polarization), and the cross-polarized $\left(\mathrm{I}_{\perp}, 90^{\circ}\right.$ with respect to the incident polarization) components of the signal. A spectrometer (SpectraPro150; Princeton Instruments, Trenton, NJ), with the slit positioned at the same angle as the incident polarization and located in the Fourier plane of the collection lens serves to spread the angular distribution into its component wavelengths. Finally, a CCD camera (VersArrayXP; Photometrics, Tucson, AZ) collects the resulting map of scattering intensity as a function of angle and wavelength. In order to account for stray light and the spectral profile of the source, a background signal was taken with no sample in the path of the incident beam, and a polytetrafluoroethylene reflectance standard was used as a sample to characterize the incident spectrum. Tissue reflectance signals were normalized by subtracting the background and then dividing by the spectrum from the reflectance standard.

\subsection{Low-coherence enhanced backscattering}

Enhanced backscattering (EBS, also known as coherent backscattering or CBS) is a self interference phenomenon arising from constructive interference of coincident photons traveling identical paths in oppo- 
site directions. Low-coherence Enhanced backscattering (LEBS) spectroscopy has recently been developed to overcome many of the limitations in conventional enhanced backscattering. The broadband light with a low spatial coherence allows for reduction of noise-like speckle, simpler measurement due to peak broadening, and the potential for obtaining diagnostic spectroscopic information. LEBS has been reported in detail in our previous publications $[8,9,11]$. Briefly, the principle of LEBS measurements can be described as a combination of two modifications to conventional EBS: broadband illumination with a low spatial coherence relative to the mean free path (spatial coherence length Lsc $\left.\ll 1 s^{\prime}\right)$ and spectrally resolved detection. Decreasing the Lsc of illumination prevents photons that emerge at large separation distances from contributing to the peak. Therefore, a smaller Lsc causes a reduction in the separation distance between photons and translates to a shorter penetration depth [23]. The combination of a short penetration depth with spectroscopic capability results in a robust and valuable tool for measuring architectural changes in epithelial tissue. Analysis of the low-coherence enhanced backscattering peak allows quantifying scattering markers that correspond to the top 30-70 $\mu \mathrm{m}$ layer of the tissue [8].

The LEBS peak was isolated by subtracting the incoherent baseline (defined as $>1^{\circ}$ away from the top of the peak) obtained by 2 nd order polynomial fit. LEBS peaks were then normalized to 0 at their incoherent baseline with the units of backscattering intensity as a ratio to the reflectance standard. LEBS signals were quantified with three markers including peak width, enhancement factor, and autocorrelation decay. The peak width was defined as the average full width at half-maximum (FWHM) in the wavelength range of 620 to $660 \mathrm{~nm}$. The values of the peak width obtained from biopsies were measured with respect to reflectance standard peak width. The LEBS enhancement factor was calculated as the average height of the LEBS peak over the same wavelength range as the LEBS width. The enhancement factor from tissue was normalized by the enhancement factor obtained from the reflectance standard in order to account for minor variations in the coherence length due to system alignment. In order to quantify the spectral fluctuations in the LEBS peak, we performed a spectral autocorrelation analysis for narrow angle averages $\left(\sim 0.05^{\circ}\right)$ in the wavelength range of $570-640 \mathrm{~nm}$. We calculated the autocorrelation function from LEBS spectra as $C(\Delta k)=\int I_{L E B S}(k) \cdot I_{L E B S}(k+\Delta k) d k$, where k is the wavenumber $(\mathrm{k}=2 \pi / \lambda)$ and found that $\mathrm{C}(\Delta \mathrm{k})$ is of the form $C(\Delta k) \propto \exp \left(-\Delta k^{2} D\right)$ for small $\Delta \mathrm{k}$, with $\mathrm{D}$ referred to as the correlation marker.

\subsection{Four-dimensional elastic light-scattering fingerprinting (4D-ELF)}

4D-ELF provides light scattering signatures in four dimensions: wavelength, scattering angle, azimuthal angle and polarization). Polarization was used to shorten the penetration depth of the optical signal $[10,14]$ to selectively collect signals primarily from epithelial tissue. Analysis of the angle and wavelength-dependent polarization gating signal can quantify tissue properties from penetration depths of $100-150 \mu \mathrm{m}[10,14]$.

We utilized two markers from the 4D-ELF polarization gating signal, spectral slope and fractal dimension, which have been demonstrated as diagnostic in earlier studies $[13,19,20]$. The spectral profile of light scattering from tissue, $\Delta \mathrm{I}(\lambda)$, is generally a declining function of wavelength, with the rate of decline being determined by properties of tissue including, refractive index variations and size distributions of structures. In general, larger concentrations of small particles will cause the spectrum to shift the dependence toward the Rayleigh scattering regime where the intensity is proportional to $\lambda^{-4}$. Alternatively, a decreased concentration of small particles will cause this dependence to be lost, resulting in a more uniform spectrum. Spectral slope is a conveniently measured marker which quantifies the declining nature of the signal and can be associated with the contribution from small-particle scattering. Additionally, the angular distribution of the polarization gating signal can be used to quantify larger structures by fractal dimension [13]. Fractal dimension was calculated as follows: the two-point mass correlation function, $\mathrm{C}(\mathrm{r})$ was calculated as the Fourier transform of the angular distribution $\Delta \mathrm{I}(\theta)$, where polarization gating signal was average within a $623-674 \mathrm{~nm}$ wavelength range. A power-law fit of $\mathrm{C}(\mathrm{r})$ was obtained in the range of 1 to $50 \mu \mathrm{m}$ in the form of $\mathrm{C}(\mathrm{r})=$ $\mathrm{r}^{\mathrm{D}-3}$, where $\mathrm{D}$ is the fractal dimension [25]. Note that the two-point mass correlation function $\mathrm{C}(\mathrm{r})$ is distinct from the spectral autocorrelation function $\mathrm{C}(\Delta \mathrm{k})$.

\subsection{Statistical analysis}

We utilized logistic regression provided by Stata version 8.0 (StataCorp LP, College Station, TX) in order to combine the 5 optical markers into a diagnostic marker. Logistic regression is an approach that constructs a diagnostic index by combining the five markers in a weighted summation: $I=\ln \left(\frac{p}{1-p}\right)=$ $\beta_{0}+\sum_{n=1}^{5} \beta_{n} O_{n}$, where $\mathrm{I}$ is the constructed index, $\mathrm{p}$ 
is the probability of a positive observation, $\mathrm{O}_{n}$ is one of the five optical markers, and $\beta_{n}$ are the coefficients determined by the algorithm. We first evenly split our data set into a training set and a testing set by assigning all odd numbered patients into the training set and all even numbered patients into the testing set. We used logistic regression to determine the values of the coefficients, and then tested the accuracy of the fit with the independent testing set by choosing a cut-off value that maximized the sensitivity and specificity of the training set. We then performed an analysis on the combined data set in order to maximize the power of further statistical analysis. All p-values were determined with a single-factor ANOVA and significance between groups was determined with a confidence level of 5\% using the Tukey-Kramer multiple comparison algorithm in Matlab 7.0 (MathWorks; Natick, MA). Unadjusted pvalues from student's t-tests were then obtained between categories to determine group-dependent differences.

\section{Results}

\subsection{Patient characteristics}

Samples from the periampullary duodenum were acquired from a total of 203 patients. Of these patients, 176 had biopsies obtained during endoscopy and 27 had biopsies taken from the surgically resected Whipple specimen, taken within 30 minutes of the procedure. The age, gender, and race distribution for periampullary biopsy patients are shown for each clinical classification in Table 1. 44 patients were diagnosed with pancreatic adenocarcinomas, of which 26 were resectable. There was 1, 4, 21, 5, and 13 cases diagnosed as stage 0 , I, II, III, IV cancer, respectively.

\subsection{Changes in low-coherence enhanced backscattering signals}

Average LEBS signals from healthy control patients and patients with pancreatic adenocarcinoma are shown in Fig. 1. (Characteristic polarization gating "fingerprints" were shown in our earlier paper). The visually assessable changes in the signal were quantified with three LEBS markers: the angular peak width, the peak enhancement factor, and the spectral correlation decay. Values of these markers from periampullary duodenal biopsies are shown in Fig. 2b. Changes in these three LEBS markers are highly significant $(p<0.0002)$ between control patients and patients with pancreatic adenocarcinoma. Changes in LEBS markers were not significant between control patients and patients with mucinous cysts (Fig. 2b, $p>0.05$ ), however, significant differences were seen for LEBS markers between patients with mucinous cysts and patients with adenocarcinoma $(p<0.05)$. As anticipated, the step-wise progression in LEBS markers mirrored the malignant transformation of pancreatic cancer.

\subsection{Changes in $4 D-E L F$ signals}

4D-ELF markers also offer valuable insight into the scattering properties of complementary microarchitecture. The values of $\Delta \mathrm{I}$ spectral slope and fractal dimension are shown in Fig. 2a. Significantly different values from control patients were found for patients with pancreatic adenocarcinoma $(p<0.05)$. The decrease in spectral slope indicates a smaller proportion of small particles (i.e. less than $\sim 200 \mathrm{~nm}$ ), while the increase in fractal dimension indicates an increase in the density of structural organization. In contrast to the LEBS markers, the 4D-ELF markers show a significant change from controls $(p<0.05)$ in patients with mucinous cysts. Therefore, 4D-ELF may be useful in identifying such early stage lesions.

\subsection{Performance characteristics}

We performed a series of statistical analysis in order to test and validate the diagnostic potential of measuring optical markers from the periampullary mucosa. As described in the methods section, we first split the data into training and testing sets. Using logistic regression to determine the coefficients of the linear combination, we were able to achieve an area under the receiver operator characteristic (ROC) curve of $81 \%$ (Table 2). A cut-off was then chosen from this ROC curve to maximize both sensitivity and specificity ( $86 \%$ and $62 \%$ respectively). By applying these coefficients and cut-off on the independent testing set, an $85 \%$ area under the receiver operator characteristic (AUROC) curve, $95 \%$ sensitivity, and $71 \%$ specificity was obtained. The results of the performances of the training and testing sets are listed in the first 2 rows of Table 2. The training and testing set were then combined to determine the coefficients more accurately, yielding an $85 \%$ AUROC, $73 \%$ sensitivity, and $89 \%$ specificity. Performances for discriminating healthy control patients and early stage (resectable) adenocarcinomas and mucinous cysts were calculated to be $88 \%$ AUROC and $79 \%$ AUROC respectively (Table 2). 
Table 1

Patient demographics

\begin{tabular}{lccc}
\hline & Age (mean \pm S.D.) & Gender (male: female) & Race (nonwhite) \\
\hline Healthy controls & $55 \pm 13$ & $27: 57$ & $78: 6$ \\
Family history & $52 \pm 9$ & $11: 13$ & $24: 0$ \\
Pancreatitis & $56 \pm 10$ & $7: 5$ & $11: 1$ \\
Mucinous cyst & $67 \pm 15$ & $7: 14$ & $20: 1$ \\
Pancreatic adenocarcinoma & $72 \pm 12$ & $15: 29$ & $40: 4$ \\
Other malignancy ${ }^{1}$ & $58 \pm 18$ & $3: 4$ & $4: 3$ \\
All patients & $60 \pm 14$ & $75: 128$ & $188: 15$ \\
\hline${ }^{1}$ Other malignancies includes 3 patients with islet cell tumors, 2 patients with pancreatic lymphoma, \\
1 cholangiocarcinoma , and 1 ampullary adenoma \\
${ }^{2}$ Includes 2 patients with pseudocysts , 8 patients with unspecified cysts, and 1 patient with chole- \\
docholithiasis
\end{tabular}

Table 2

Performance characteristics

\begin{tabular}{lccc}
\hline Sensitivity & Specificity & AUROC & \\
\hline Training set (odd \# patients) & $86 \%$ & $62 \%$ & $81 \%$ \\
Testing set (even \# patients) & $95 \%$ & $71 \%$ & $85 \%$ \\
Healthy control vs. pancreatic adenocarcinoma) & $73 \%$ & $89 \%$ & $85 \%$ \\
Healthy control vs. resectable adenocarcinoma) & $73 \%$ & $89 \%$ & $88 \%$ \\
Healthy control vs. mucinous cyst & $71 \%$ & $77 \%$ & $79 \%$ \\
\hline
\end{tabular}

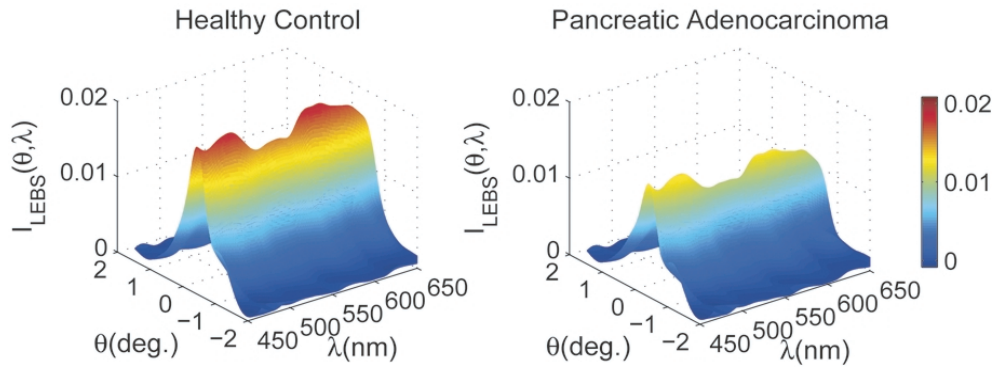

Fig. 1. Average LEBS signals from periampullary duodenal mucosa from healthy control patients and patients with pancreatic adenocarcinoma. The grayscale is representative of the LEBS signal intensity, also corresponding to the vertical axis. The other two axes represent the angle and the wavelength of backscattered light. The differences in the LEBS signals are quantified with 3 parameters including the angular full width at half maximum, the height of the peak (enhancement), and spectral autocorrelation decay.

\subsection{Optical markers in higher risk population groups}

In our cohort of biopsied patients, we acquired LEBS/4D-ELF data from 24 patients with a family history of pancreatic cancer and 12 patients with pancreatitis (4 acute pancreatitis). These groups are at a higher risk of developing pancreatic cancer than the general population and therefore could potentially be the initial target population for a screening procedure. Figure 3 shows results of the Tukey-Kramer test, where non-overlapping lines within a group indicate statistical significance at the $95 \%$ confidence level. In general, these higher risk conditions tend to fall within the range of marker values between healthy control patients and pancreatic adenocarcinoma patients. Although there are significant differences seen in LEBS enhancement factor and $\Delta \mathrm{I}$ spectral slope, a longitu- dinal study would be required to determine which of these patients are exhibiting the pre-malignant phenotype which would later result in pancreatic cancer. The top left panel in Fig. 3 is a comparison chart showing the mean and $95 \%$ confidence intervals of these groups for a probability index constructed from these optical markers when combined in a linear combination that is optimized with logistic regression to distinguish the difference between healthy control and pancreatic cancer. Although there were too few cases to make statistical conclusions for other pancreatic malignancies, islet cell tumor cases had a probability index of 0.66 \pm 0.18 , pancreatic lymphoma cases had a probability index of $0.37 \pm 0.08$ (mean \pm std. dev.), and the 1 cholangiocarcinoma patient had a probability index of 0.70. Additionally, two cases with benign pseudocysts 


\section{$\square$ Healthy Controls $\square$ Patients with mucinous cysts $\square$ Patients with adenocarcinomas}

A
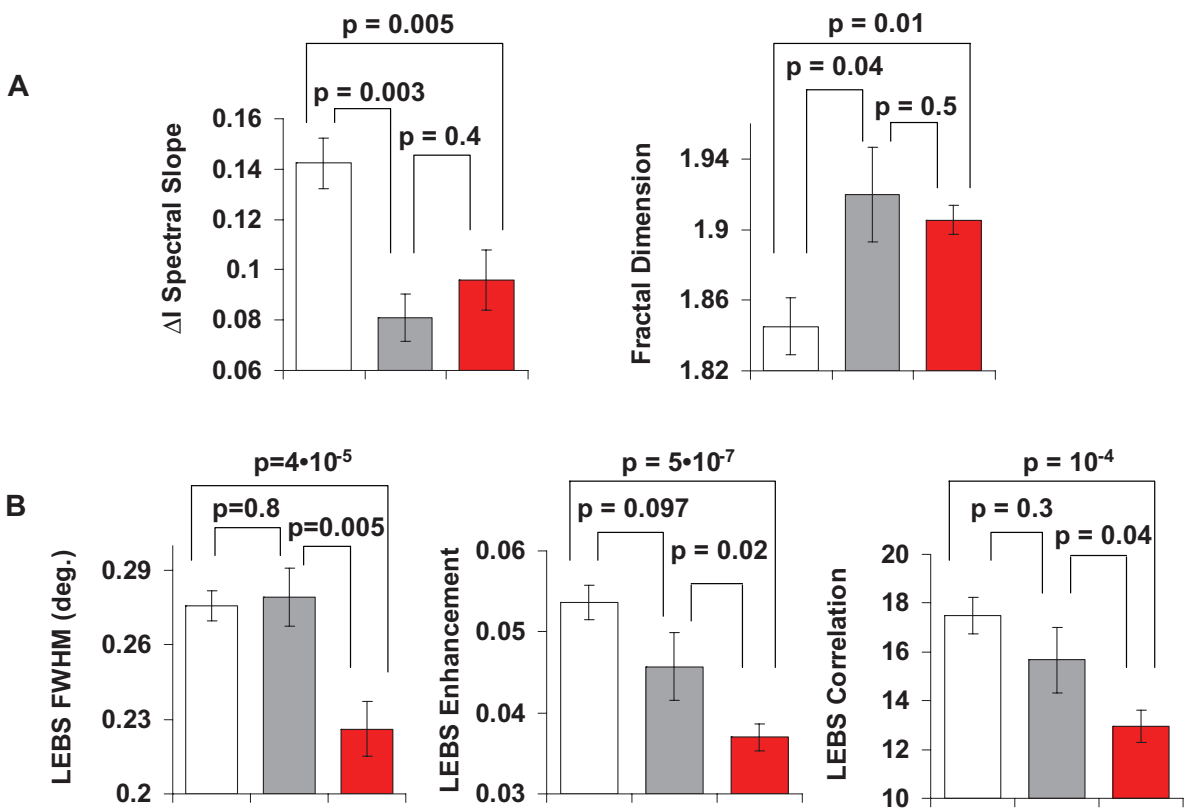

Fig. 2. Values of 4D-ELF and LEBS optical parameters as measured from endoscopically normal duodenal periampullary mucosa exhibit significant trends with the development of cancer. In A, 4D-ELF parameters $\Delta \mathrm{I}$ spectral slope and fractal dimension in control patients have significantly different values from pancreatic cancer patients and patients with mucinous cysts $(p<0.05)$. In B, LEBS FWHM, LEBS enhancement, and LEBS autocorrelation decay rate exhibit significant differences between control and cancer patients $\left(p=4 \cdot 10^{-5}, 5 \cdot 10^{-7}\right.$, and $10^{-4}$ respectiviely). Although marginally significant differences were seen in LEBS enhancement between control patients and patients with mucinous cysts $(p=0.097)$, LEBS parameters revealed significant differences between patients with mucinous cysts and patients with cancer $(p<0.05)$

had a probability index of $0.27 \pm 0.009$ (mean \pm std. dev.).

\section{Discussion and conclusions}

The detection and screening of pancreatic cancer presents many challenges; some of which can not be addressed with existing technologies. Optical surveillance of the duodenal tissue adjacent to the pancreas is one approach which is simple yet sensitive enough to potentially be used as a screening technique. In this study, we have shown that LEBS and 4D-ELF optical markers quantified from the duodenal periampullary mucosa are capable of detecting pancreatic adenocarcinoma. A $95 \%$ sensitivity, $71 \%$ specificity and $85 \%$ AUROC was achieved from an independent testing set for discriminating between healthy patients and patients with pancreatic adenocarcinoma. Importantly, it appeared that the stage of disease did not impact the results with an $88 \%$ AUROC obtained for detecting patients in resectable stages of cancer (stage 0-2). We were also able to demonstrate the ability to discrimi- nate mucinous cyst lesions, which are precursors of the disease, from healthy controls (Table 2).

Although both LEBS and 4D-ELF markers from periampullary biopsies show significant differences between healthy control and pancreatic adenocarcinoma patients (Fig. 2), these markers have varying sensitivities to early-stage precursors of the disease. Interestingly, LEBS markers are highly diagnostic for pancreatic cancer $\left(p<10^{-4}\right)$, while being relatively insensitive to the presence of precancerous mucinous lesions ( $p=$ 0.97 for LEBS enhancement, $p>0.1$ for LEBS FWHM and LEBS correlation decay). On the other hand, 4DELF markers exhibit sensitivity to the presence of these pre-cancerous lesions $(p<0.05)$. One possibility for this difference is that changes in pre-cancerous events exhibit different depth-related changes in the duodenal tissue. The LEBS signal consists of photons that penetrate $40-70 \mu \mathrm{m}$ of the duodenal mucosa, while the polarization gating signal penetrates approximately $100 \mu \mathrm{m}$, therefore evaluating a different tissue layer. Therefore, the two methods are complimentary in sensing pre-malignant phenotypes indicative of early and later stages of pancreatic cancer development. 


\begin{tabular}{|llllrr|}
\hline HC & H ealthy Control & FH & F amily History & PT & Pancreatitis \\
MC & M ucinous Cyst & PA & P ancreatic Adenocarcinoma & \\
\hline
\end{tabular}
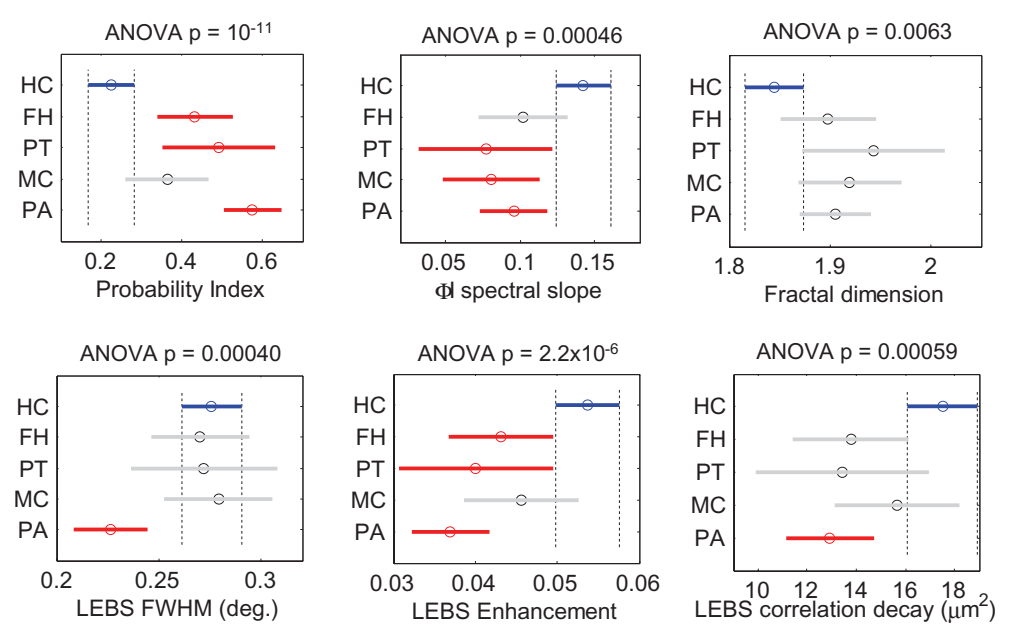

Fig. 3. Multiple comparison plots of combined probability index and the 5 optical parameters as measured from the periampullary duodenum. ANOVA p-values of $<0.05$ indicate significance within the data set. The multiple comparisons tests are performed to determine which groups are significantly different from each other by accounting for the variability of the entire data set. The horizontal lines represent $95 \%$ confidence intervals of the mean, and when non-overlapping signify that the two groups are significantly different $(p<0.05)$. Groups that are significantly different from controls are shown in red. Patients with a family history of pancreatic cancer exhibited significant differences from controls as measured by LEBS enhancement. Patients with pancreatitis showed significant differences from control in LEBS enhancement and $\Delta \mathrm{I}$ spectral slope. Both groups were significantly different when compared as the combined probability index.

Patients with a family history of pancreatic cancer have significantly higher risk of developing the malignancy and are a good potential target for a screening population. We evaluated duodenal biopsies from patients in these higher risk groups and found that two of the five optical markers, LEBS enhancement and $\Delta \mathrm{I}$ spectral slope, were significantly shifted toward cancer $(p<0.05)$. Therefore, the phenotype of individuals with a strong family history, as measured by these two markers, may be shifted towards that of patients with cancer. However, many of these individuals will never develop the disease and a longitudinal study is needed in order to determine the potential of LEBS and 4D-ELF optical markers for identifying these patients.

In addition to considering screening of a higher risk population, it is also important to identify benign inflammatory conditions of the pancreas. Often focal pancreatitis can be difficult to discriminate from an underlying pancreatic cancer, so an optical evaluation of the periampullary duodenum that excludes cancer would be of clinical use. Complicating this assessment is the fact that chronic pancreatitis is a recognized risk factor for pancreatic cancer [15]. Our preliminary findings in 12 patients indicate that the signal from this group lies between cancer and healthy control patients, and in most optical markers is very close to the family history group (Fig. 3).
Other pancreatic malignancies, although less common, are also important to identify and diagnose in order to determine an appropriate treatment. We observed 7 patients with other pancreatic malignancies, of which, the two largest types were islet cell tumors (3 patients) and pancreatic lymphoma (2 patients). Although these numbers were too few for statistically meaningful conclusions, the value of the combined probability index for the islet cell tumor group, $0.66 \pm 0.18$ (mean \pm std. dev.), was close to that of pancreatic adenocarcinoma, while the values of the pancreatic lymphoma patients were between the control and adenocarcinoma group: $0.37 \pm 0.08$ (mean \pm std. dev.). For comparison, measurements obtained from 2 patients with benign pseudocysts appropriately showed probability index values close to those of control patients $(0.27 \pm 0.009)$. More patient data is required to determine whether our optical approach can distinguish mucinous cysts, which have malignant potential from benign cystic lesions that do not have malignant potential.

Although the data were not shown, it is possible to perform 4D-ELF and LEBS measurements in a retrospective fashion on archived tissue specimens. Obtaining these types of measurements was labor intensive and technically challenging but, as expected, a difference was also seen in the signals between cancer and control. Furthermore, the optical signals obtained were 
clearly different than those obtained on fresh tissue, thereby strongly limiting their use clinically beyond feasibility studies.

In summary, LEBS and 4D-ELF derived optical markers demonstrate great potential for detecting the field effect of pancreatic cancer. The evaluation of a testing set resulted in a $95 \%$ sensitivity, $71 \%$ specificity, and $81 \%$ AUROC for discriminating between healthy control patients and patients with pancreatic adenocarcinoma by evaluating tissue from the periampullary duodenum. Furthermore, the performance was uncompromised when these markers were evaluated for the detection of early, potentially curable, stages of pancreatic cancer. These optical markers also demonstrate potential in detecting precursor mucinous cystic lesions. Other facets of the diagnostic potential of LEBS and 4D-ELF markers may include screening higher risk populations with a family prevalence of pancreatic cancer, or the ability to discern patients with benign inflammatory conditions from patients with pancreatic cancer.

\section{Acknowledgements}

This study was supported by the V Foundation, Rolfe Foundation, NSF grant CBET-0733868, and NIH grants R21 EB006742, R01CA112315, and R01 EB003682.

\section{References}

[1] R.E. Brand, M.M. Lerch, W.S. Rubinstein, J.P. Neoptolemos, D.C. Whitcomb, R.H. Hruban, T.A. Brentnall, H.T Lynch, M.I. Canto and P.t. ISIDP, Advances in counselling and surveillance patients at risk for pancreatic cancer, Gut $\mathbf{5 6}$ (2007), 1460-1469.

[2] L.C. Chen, C.Y. Hao, Y.S.Y. Chiu, P. Wong, J.S. Melnick, M. Brotman, J. Moretto, F. Mendes, A.P. Smith, J.L. Bennington, D. Moore and N.M. Lee, Alteration of gene expression in normal-appearing colon mucosa of $\mathrm{APC}(\mathrm{min})$ mice and human cancer patients, Cancer Research 64 (2004), 3694-3700.

[3] K.C. Conlon, Intraductal papillary mucinous tumors of the pancreas, Journal of Clinical Oncology 23 (2005), 4518-4523.

[4] A.L. Cubilla and P.J. Fitzgerald, Morphological Lesions Associated with Human Primary Invasive Nonendocrine Pancreas Cancer, Cancer Research 36 (1976), 2690-2698.

[5] W.A. Franklin, A.F. Gazdar, J. Haney, I.I. Wistuba, F.G. LaRosa, T. Kennedy, D.M. Ritchey and Y.E. Miller, Widely dispersed P53 mutation in respiratory epithelium - A novel mechanism for field carcinogenesis, Journal of Clinical Investigation 100 (1997), 2133-2137.

[6] M.Z. Guo, M.G. House, C. Hooker, Y. Han, E. Heath, E. Gabrielson, S.C. Yang, S.B. Baylin, J.G. Herman and M.V. Brock, Promoter hypermethylation of resected bronchial margins: A field defect of changes, Clinical Cancer Research $\mathbf{1 0}$ (2004), 5131-5136.
[7] A. Jemal, R. Siegel, E. Ward, Y.P. Hao, J.Q. Xu, T. Murray and M.J. Thun, Cancer statistics, Ca-a Cancer Journal for Clinicians 58 (2008), 71-96.

[8] Y.L. Kim, Y. Liu, V.M. Turzhitsky, R.K. Wali, H.K. Roy and V. Backman, Depth-resolved low-coherence enhanced backscattering, Optics Letters 30 (2005), 741-743.

[9] Y.L. Kim, Y. Liu, R.K. Wali, H.K. Roy and V. Backman, Lowcoherent backscattering spectroscopy for tissue characterization, Applied Optics 44 (2005), 366-377.

[10] Y.L. Kim, Y. Liu, R.K. Wali, H.K. Roy, M.J. Goldberg, A.K. Kromin, K. Chen and V. Backman, Simultaneous measurement of angular and spectral properties of light scattering for characterization of tissue microarchitecture and its alteration in early precancer, Ieee Journal of Selected Topics in Quantum Electronics 9 (2003), 243-256.

[11] Y.L. Kim, V.M. Turzhitsky, Y. Liu, H.K. Roy, R.K. Wali, H. Subramanian, P. Pradhan and V. Backman, Low-coherence enhanced backscattering: review of principles and applications for colon cancer screening, Journal of Biomedical Optics 11 (2006).

[12] W. Kimura, H. Nagai, A. Kuroda, T. Muto and Y. Esaki, Analysis of small cystic lesions of the pancreas, International Journal of Pancreatology 18 (1995), 197-206.

[13] Y. Liu, R.E. Brand, V. Turzhitsky, Y.L. Kim, H.K. Roy, N. Hasabou, C. Sturgis, D. Shah, C. Hall and V. Backman, Optical markers in duodenal mucosa predict the presence of pancreatic cancer, Clinical Cancer Research 13 (2007), 4392-4399.

[14] Y. Liu, Y.L. Kim, X. Li and V. Backman, Investigation of depth selectivity of polarization gating for tissue characterization, Optics Express 13 (2005), 601-611.

[15] A.B. Lowenfels, P. Maisonneuve, E.P. DiMagno, Y. Elitsur, L.K. Gates, J. Perrault, D.C. Whitcomb, G. Aranha, P. Banks, F.R. Burton, D. CarrLocke, W.P. Dyck, R.G. Gish, R.L. Goodale, G. Lehman, S.P. Martin, J. Potts, S. Sherman, C.D. Ulrich, P. Yakshe, P. Yeaton, Y. Hamanaka, M. Koizumi, T. Tomioka, T. Tsunoda, K. Yamadera, J.P. Delmont, H.G. Beger, A. Holstege, V. Keim, P. Layer, J. Triantafillidis, P. Boyle, G. Cavallini, L. Gullo, S. Pedrazzoli, G. Uomo, D.G.L. Castano, I. Ihse, M. Buchler and E. Elias, Hereditary pancreatitis and the risk of pancreatic cancer, Journal of the National Cancer Institute 89 (1997), 442-446.

[16] H. Matsubayashi, M. Canto, N. Sato, A. Klein, T. Abe, K. Yamashita, C.J. Yeo, A. Kalloo, R. Hruban and M. Goggins, DNA methylation alterations in the pancreatic juice of patients with suspected pancreatic disease, Cancer Research 66 (2006), 1208-1217.

[17] H. Matsubayashi, N. Sato, K. Brune, A.L. Blackford, R.H. Hruban, M. Canto, C.J. Yeo and M. Goggins, Age- and disease-related methylation of multiple genes in nonneoplastic duodenum and in duodenal juice, Clinical Cancer Research 11 (2005), 573-583.

[18] H.K. Roy, Y.L. Kim, Y. Liu, R.K. Wali, M.J. Goldberg, V. Turzhitsky, J. Horwitz and V. Backman, Risk stratification of colon carcinogenesis through enhanced backscattering spectroscopy analysis of the uninvolved colonic mucosa, Clinical Cancer Research 12 (2006), 961-968.

[19] H.K. Roy, Y.L. Kim, R.K. Wali, Y. Liu, J. Koetsier, D.P. Kunte, M.J. Goldberg and V. Backman, Spectral markers in preneoplastic intestinal mucosa: An accurate predictor of tumor risk in the MIN mouse, Cancer Epidemiology Biomarkers \& Prevention 14 (2005), 1639-1645.

[20] H.K. Roy, Y. Liu, R.K. Wali, Y.L. Kim, A.K. Kromine, M.J. Goldberg and V. Backman, Four-dimensional elastic lightscattering fingerprints as preneoplastic markers in the rat 
model of colon carcinogenesis, Gastroenterology 126 (2004), 1071-1081.

[21] M. Singh and A. Maitra, Precursor lesions of pancreatic cancer: Molecular pathology and clinical implications, Pancreatology 7 (2007), 9-19.

[22] A. Spira, J.E. Beane, V. Shah, K. Steiling, G. Liu, F. Schembri, S. Gilman, Y.M. Dumas, P. Calner, P. Sebastiani, S. Sridhar, J. Beamis, C. Lamb, T. Anderson, N. Gerry, J. Keane, M.E. Lenburg and J.S. Brody, Airway epithelial gene expression in the diagnostic evaluation of smokers with suspect lung cancer, Nature Medicine 13 (2007), 361-366.

[23] H. Subramanian, P. Pradhan, Y.L. Kim and V. Backman, Penetration depth of low-coherence enhanced backscattered light in subdiffusion regime, Physical Review E 75 (2007).

[24] A.C. Tersmette, G.M. Petersen, G.J.A. Offerhaus, F.C.
Falatko, K.A. Brune, M. Goggins, E. Rozenblum, R.E. Wilentz, C.J. Yeo, J.L. Cameron, S.E. Kern and R.H. Hruban, Increased risk of incident pancreatic cancer among first-degree relatives of patients with familial pancreatic cancer, Clinical Cancer Research 7 (2001), 738-744.

[25] A. Wax, C.H. Yang, V. Backman, K. Badizadegan, C.W. Boone, R.R. Dasari and M.S. Feld, Cellular organization and substructure measured using angle-resolved low-coherence interferometry, Biophysical Journal 82 (2002), 2256-2264.

[26] J.M. Winter, J.L. Cameron, K.D. Lillemoe, K.A. Campbell, D. Chang, T.S. Riall, J. Coleman, P.K. Sauter, M. Canto, R.H. Hruban, R.D. Schulick, M.A. Choti and C.J. Yeo, Periampullary and pancreatic incidentaloma a single institution's experience with an increasingly common diagnosis, Annals of Surgery 243 (2006), 673-683. 


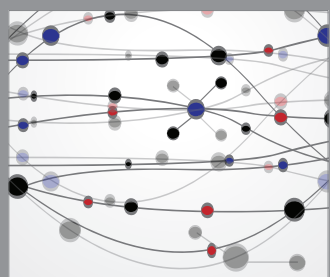

The Scientific World Journal
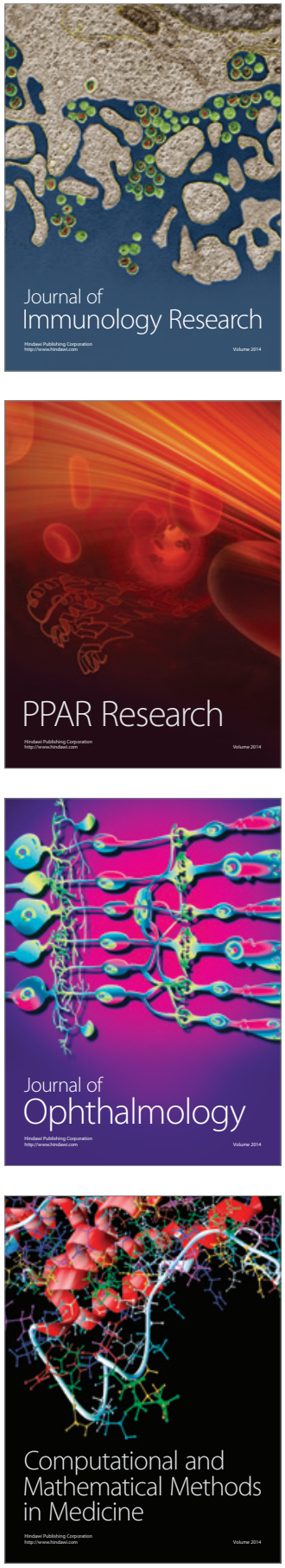

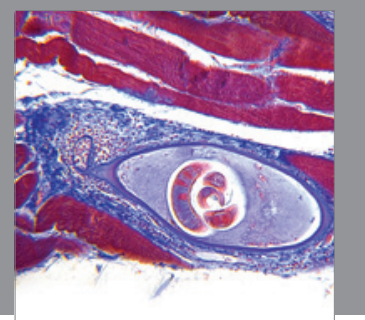

Gastroenterology

Research and Practice
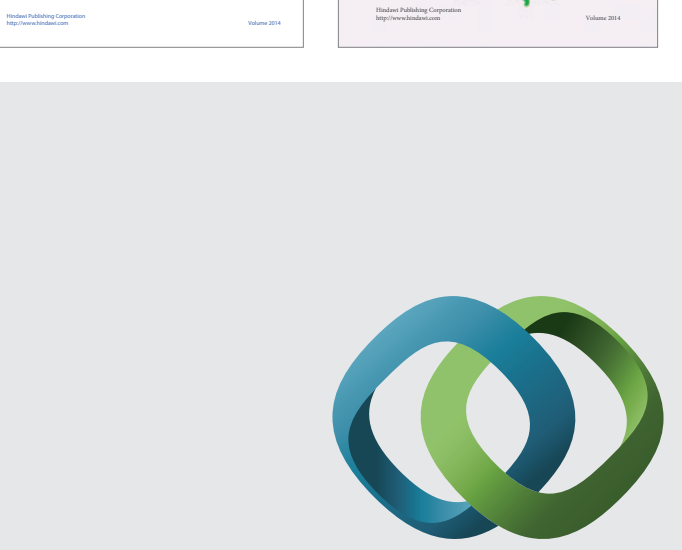

\section{Hindawi}

Submit your manuscripts at

http://www.hindawi.com
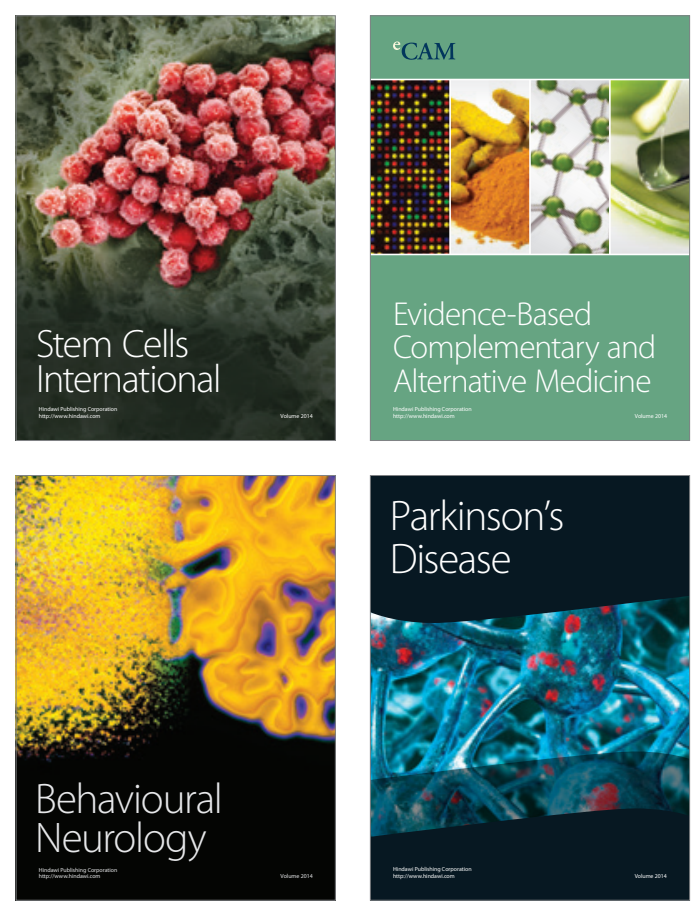

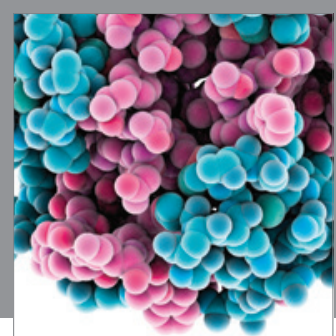

Journal of
Diabetes Research

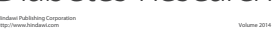

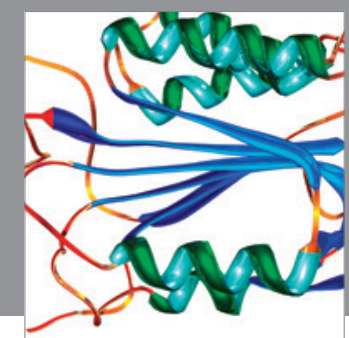

Disease Markers
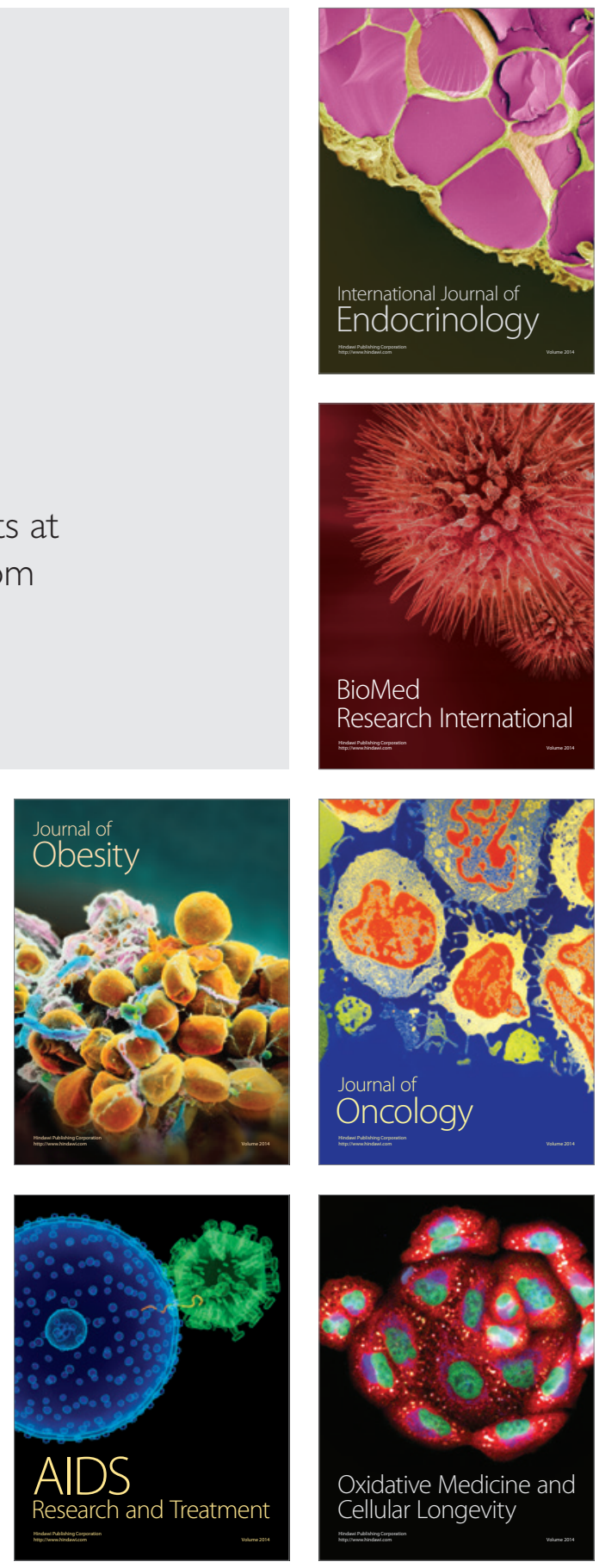